\title{
Abordagem multivariada para endogamia e valor fenotípico utilizando diferentes estratégias de cruzamento ${ }^{1}$
}

\author{
Marcelo Jangarelli
}

\begin{abstract}
Multivariate approach to endogamy and phenotypic value using different crossbreeding strategies

Simulation has contributed to the advance of genetic improvement. This study aimed at evaluating selective mating by using the distribution of extremes to maximize the phenotypic increment and slow down the endogamy increment, under a multivariate approach. Simulated data were used to assess mating strategies in different family sizes, over 20 generations. The cluster analysis made possible to distinguish the selective mating from other strategies for each family size. By combining mating strategy and family size, the cluster analysis pointed out the superiority of the selective mating to optimize the phenotypic value and slow down the endogamy increment. The cluster methods showed equivalence in the formation of groups with homogeneous strategies for the estimates of phenotypic mean values and mean endogamy. The multivariate analysis corroborated superior phenotypic increments to the selective mating, as well as lower endogamy levels, over the generations.
\end{abstract}

KEY-WORDS: Selective mating; cluster analysis; marker assisted selection.

\section{INTRODUÇÃO}

Avanços têm ocorrido em programas de melhoramento genético, por meio da utilização de indivíduos (plantas e animais) geneticamente superiores (Neves et al. 2009, Ishii et al. 2013). Neste sentido, estratégias de acasalamento e de seleção permitem acelerar o progresso genético e dinamizar a difusão de material genético superior. Figuram-se, neste cenário, acasalamentos estratégicos que combinam indivíduos similares ou não, considerando-se o fenótipo ou genótipo como critério de semelhança (Neves et al. 2009).

A genotipagem seletiva é uma estratégia capaz de otimizar o poder de detecção de locos de caracteres

\section{RESUMO}

A simulação tem contribuído para o avanço do melhoramento genético. Este estudo objetivou avaliar o acasalamento seletivo, utilizando a distribuição dos extremos para maximizar o incremento fenotípico e retardar o acréscimo da endogamia, por meio de abordagem multivariada. Dados simulados foram utilizados para avaliar estratégias de acasalamento, em diferentes tamanhos de família, no decorrer de 20 gerações. A análise de agrupamento possibilitou diferenciar o acasalamento seletivo das demais estratégias, em cada tamanho de família. Combinando-se estratégia de acasalamento e tamanho de família, a análise de agrupamento assinalou superioridade do acasalamento seletivo, na capacidade de otimizar o valor fenotípico e retardar o incremento da endogamia. Os métodos de agrupamento apresentaram equivalência na formação de grupos com estratégias homogêneas, para as estimativas dos valores fenotípicos médios e endogamia média. A análise multivariada corroborou incrementos fenotípicos superiores, para o acasalamento seletivo, bem como menores níveis endogâmicos, ao longo das gerações.

PALAVRAS-CHAVE: Acasalamento seletivo; análise de agrupamento; seleção assistida por marcadores.

quantitativos, por permitir o uso racional da variabilidade genética entre os indivíduos (Rosa 2007, Zhao et al. 2012). Ela utiliza a distribuição dos extremos, genotipando somente os indivíduos presentes nos extremos superior e inferior de uma distribuição dos valores fenotípicos, de uma população experimental disponível (Lu et al. 2013).

A intensificação na utilização de métodos e técnicas que permitem disseminar material genético superior tem contribuído não apenas para acelerar o progresso genético, mas, também, para o acréscimo dos níveis endogâmicos na população, o que minimiza o incremento fenotípico a ser obtido ao longo das gerações sob seleção (Malhado et al. 2009, Reis et al. 2009, Jacometo et al. 2010). Estratégias de

1. Trabalho recebido em mar./2013 e aceito para publicação em mar./2014 (nº registro: PAT 23247).

2. Universidade Federal Rural do Rio de Janeiro (UFRRJ), Instituto de Ciências Exatas, Departamento de Matemática, Seropédica, RJ, Brasil.E-mail: gmejanga@hotmail.com. 
acasalamento podem contribuir para maximizar o valor fenotípico, devido à manutenção de maior variabilidade genética na população e ao consequente retardo nos níveis endogâmicos.

$\mathrm{Na}$ tentativa de avaliar e identificar estratégias de acasalamento similares, pode-se empregar métodos de agrupamento que permitam classificar unidades amostrais em categorias similares. As estimativas dos parâmetros genéticos e fenotípicos podem ser utilizadas como medidas de variáveis classificatórias, possibilitando o agrupamento de unidades (estratégias, por exemplo) que resultem em estimativas semelhantes dos parâmetros considerados.

Objetivou-se, com este trabalho, avaliar o acasalamento seletivo, utilizando a distribuição dos extremos, entre outras estratégias de acasalamento, na capacidade de otimizar o valor fenotípico e retardar o incremento da endogamia, por meio da aplicação da análise multivariada de agrupamento.

\section{MATERIAL E MÉTODOS}

Os procedimentos de simulação e análise de dados foram realizados de janeiro a dezembro de 2012, na Universidade Federal Rural do Rio de Janeiro (UFRRJ), em Seropédica (RJ).

Foram utilizados dados simulados pelo programa de simulação genética Genesys (Genetic System, versão 2009) (Euclydes 2009). Este programa, escrito na linguagem de programação Fortran, permite a simulação de genomas complexos, o que possibilita o estudo de populações, seguindo-se pressuposições genéticas e estatísticas de interesse.

Foi simulado um genoma hipotético, semelhante ao de aves (frango de corte), constituído de uma característica quantitativa com herdabilidade de 0,40 , a partir do qual se obtiveram as populações base e inicial. O genoma proposto estava caracterizado geneticamente (apresentava 958 centiMorgan - cM de extensão); os marcadores moleculares foram dispostos, estrategicamente, a cada $5 \mathrm{cM}$, o que totalizou 191 marcadores e 200 locos quantitativos associados à característica, distribuídos ao longo de 40 cromossomos de tamanho aleatório; os efeitos aditivos dos locos quantitativos foram simulados seguindo-se a distribuição normal dos dados fenotípicos; os locos quantitativos foram dialélicos e não possuíam desvios de dominância e nem epistasia; os efeitos de ambiente foram simulados conforme a distribuição normal; e os dados fenotípicos simula- dos apresentaram média de 10,00 unidades e desvio padrão de 2,00 unidades.

Para a estrutura genômica simulada, foi construída uma população base composta de 500 machos e 500 fêmeas, não aparentados entre si. Com os 1.000 descendentes escolhidos aleatoriamente na população base, obtidos do cruzamento de 100 machos e 100 fêmeas (uma fêmea/macho), produzindo 10 filhos/fêmea/macho (1.000 indivíduos), formou-se a população inicial. Esta população foi submetida à seleção assistida por marcadores, por 20 gerações consecutivas, com 20 repetições, visando a minimizar os efeitos da flutuação genética. O número de gerações (20) visou a atender inferências a curto, médio e longo prazo, em um programa de melhoramento genético (Leão et al. 2013).

A utilização de marcadores moleculares em programas de melhoramento implementados pela seleção assistida por marcadores pode beneficiar os processos de seleção, quando se admitem características de baixa e média herdabilidade, em que o ambiente tem considerável participação na expressão fenotípica (Rodrigues et al. 2010, Sousa et al. 2011). A seleção foi conduzida com a finalidade de incrementar o valor fenotípico.

A partir da população inicial, os reprodutores foram selecionados com base em seus genótipos, de acordo com o número de marcadores moleculares identificados que estariam estatisticamente associados aos locos quantitativos. Desta forma, os genitores eleitos em cada geração representavam os indivíduos detentores de maior informatividade dos marcadores, ou seja, aqueles que apresentavam maior número de marcadores ligados aos locos quantitativos.

A cada geração, os dez machos e as dez fêmeas (uma fêmea/macho) que obtiveram o maior número de marcadores informativos foram acasalados, adotando-se o esquema de acasalamento de um genitor masculino com apenas uma fêmea, o que assegura somente famílias de irmãos completos.

O número de progênies, em cada cruzamento, foi dependente do tamanho de família admitido $(10,20$, 30,40 e 50), o que correspondeu a 10, 20, 30, 40 e 50 descendentes, em dez acasalamentos, respectivamente. Estes descendentes formavam a geração seguinte.

Nesse contexto, as populações selecionadas tiveram tamanhos de 100, 200, 300, 400 e 500 indivíduos por geração, correspondendo a 10, 20,30, 40 e 50 progênies por casal, respectivamente. A definição dos tamanhos de família (números de progênies) 
buscou atender espécies com menor número de progênies (suínos, por exemplo), bem como espécies em que exista a possibilidade de um maior número de descendentes (aves, por exemplo).

$\mathrm{Na}$ seleção assistida por marcadores, foram comparadas três estratégias de acasalamento, em cada um dos cinco tamanhos de família mencionados, o que totalizou quinze combinações (estratégias) analisadas.

Avaliou-se o acasalamento seletivo entre os genitores, seguindo-se o princípio da genotipagem seletiva (distribuição dos extremos). Nesta estratégia, os machos e as fêmeas selecionados foram ordenados separadamente, com base no número de marcadores associados aos locos quantitativos (informatividade dos marcadores). $\mathrm{O}$ acasalamento foi realizado entre os indivíduos posicionados nos extremos opostos. Procedeu-se ao acasalamento entre os machos com os melhores desempenhos, com relação à identificação de marcadores ligados aos locos quantitativos, referidos como os melhores (localizados no extremo superior de sua classificação), e as fêmeas com os piores desempenhos na identificação de marcadores, referidas como as piores (localizadas no extremo inferior de sua classificação), e vice-versa (piores machos versus melhores fêmeas). Esta estratégia favorece a variabilidade genética, por acasalar indivíduos mais contrastantes geneticamente (Rosa 2007).

Outras duas estratégias foram comparadas. $\mathrm{Na}$ primeira, entre os indivíduos selecionados, acasalaram-se os machos e as fêmeas com melhores desempenhos na identificação de marcadores e, também, os machos e as fêmeas com desempenhos inferiores na detecção de marcadores associados aos locos quantitativos, mencionados como os piores. Nesta estratégia, existe uma tendência de acasalar indivíduos geneticamente semelhantes e, consequentemente, comprometer a variabilidade genética para o melhoramento (Zhao et al. 2012). Na segunda estratégia, os reprodutores selecionados foram acasalados aleatoriamente.

$\mathrm{O}$ número de genitores selecionados (dez machos e dez fêmeas) foi mantido, ao longo das gerações, sob seleção assistida por marcadores, o que estabelece razão sexual (d) de uma unidade (1), conforme a estrutura populacional definida (Tabela 1).

O tamanho efetivo da população $\left(\mathrm{N}_{\mathrm{e}}\right)$ foi calculado com base no estimador proposto por Wright (1931):

$$
\frac{1}{N_{e}}=\frac{1}{4 N_{m s}}+\frac{1}{4 N_{f s}}
$$

Tabela 1. Estrutura das populações sob seleção assistida por marcadores (Seropédica, RJ, 2012).

\begin{tabular}{rrrrcccc}
\hline TF & TP & $\mathrm{N}_{\mathrm{m} / \mathrm{f}}$ & $\mathrm{d}$ & $\mathrm{N}_{\mathrm{m} / \mathrm{fs}}$ & $\mathrm{P}_{\mathrm{ms} / \mathrm{fs}}$ & $\mathrm{i}_{\mathrm{m} / \mathrm{f}}$ & $\mathrm{N}_{\mathrm{e}}$ \\
\hline 10 & 100 & 50 & 1 & 10 & 0,200 & 1,40 & 20 \\
20 & 200 & 100 & 1 & 10 & 0,100 & 1,76 & 20 \\
30 & 300 & 150 & 1 & 10 & 0,067 & 1,94 & 20 \\
40 & 400 & 200 & 1 & 10 & 0,050 & 2,06 & 20 \\
50 & 500 & 250 & 1 & 10 & 0,040 & 2,15 & 20 \\
\hline
\end{tabular}

TF: tamanho da família; TP: tamanho da população; $\mathrm{N}_{\text {mf }}$ : número de machos/ fêmeas; d: valor da razão sexual; $\mathrm{N}_{\mathrm{m} / \mathrm{f}}$ : número de machos/fêmeas selecionados; $\mathrm{P}_{\mathrm{m} / \mathrm{fs}}$ : proporção de machos/fêmeas selecionados; $\mathrm{i}_{\mathrm{mff}}$ intensidade de seleção aplicada aos machos/fêmeas; $\mathrm{N}_{\mathrm{e}}$ : tamanho efetivo da população.

Em cada tamanho de família, formaram-se três populações, totalizando 15 populações, cada qual representando a combinação de uma estratégia de acasalamento e um determinado tamanho de família. Procedeu-se à seleção assistida por marcadores, em cada população, separadamente, o que resultou em 15 seleções, todas partindo do mesmo valor fenotípico (10 unidades).

O programa de simulação genética Genesys utiliza o método da marca simples para identificar associações entre marcadores e locos quantitativos, na seleção assistida por marcadores moleculares. Este método verifica a associação entre cada marcador e a característica de interesse, por meio da análise de regressão linear entre os genótipos dos marcadores e os valores fenotípicos dos descendentes dos acasalamentos. Adotou-se o nível de significância de 0,05, na regressão linear simples.

Para comparação das estratégias de acasalamento adotadas na seleção assistida por marcadores, foram estimadas as médias dos valores fenotípicos e dos coeficientes de endogamia, em cada geração. O coeficiente de endogamia (F) foi estimado pela relação entre o número de locos com um dos alelos fixados $\left(\mathrm{N}_{\mathrm{f}}\right)$ e o número total de locos $(\mathrm{N})$ :

$$
F=\frac{N_{f}}{N}
$$

Na tentativa de reduzir os problemas de colinearidade entre as gerações, os valores fenotípicos e de endogamia estimados foram padronizados e aplicados em dois métodos de agrupamento (método hierárquico da ligação completa e método de otimização de Tocher), com o propósito de identificar e validar uma estrutura classificatória entre as estratégias de acasalamento (Jangarelli et al. 2010). 
A aplicação da análise de agrupamento foi processada, separadamente, nas médias estimadas de cada parâmetro (valor fenotípico e endogamia). As 15 estratégias de acasalamento resultantes da combinação dos cinco tamanhos de família e das três estratégias de acasalamento representavam os tratamentos (Tabela 2). Cada geração sob seleção assistida por marcadores representou uma variável, o que totalizou 20 variáveis. As médias obtidas nestas variáveis foram utilizadas com a finalidade de reunir estratégias de acasalamento similares, que apresentavam semelhança no padrão de comportamento/ resposta, em relação às gerações sob seleção.

A distância euclidiana média foi utilizada em ambos os métodos (hierárquico e otimização), para quantificar as dissimilaridades entre os tratamentos (estratégias de acasalamento), pois ela não é influenciada pelo número de variáveis, além de as estimativas dos parâmetros, em cada geração, representarem um único valor médio de 20 repetições.

Para as análises multivariadas de agrupamento, em cada parâmetro genético estimado, foi utilizado o Sistema para Análises Estatísticas e Genéticas (SAEG), descrito por Ribeiro Júnior \& Melo (2008).

\section{RESULTADOS E DISCUSSÃO}

Os valores fenotípicos evidenciaram superioridade no ganho, ao longo das gerações, sob seleção assistida por marcadores, ao adotar o acasalamento seletivo entre os melhores e os piores genitores selecionados (distribuição dos extremos - MP), em analogia às outras duas estratégias (acasalamento ao acaso - AA e acasalamento entre os melhores - MM) (Tabela 2).

Independentemente do tamanho de família, maiores progressos fenotípicos foram obtidos com o acasalamento seletivo, apesar de famílias com dez descendentes demonstrarem menor intensidade na ascensão do incremento fenotípico, embora sempre superior aos demais acasalamentos.

A distribuição dos extremos (MP) requer menor número de gerações sob seleção, para que os ganhos fenotípicos sejam equivalentes aos das estratégias cujo acasalamento foi aleatório ou entre os melhores. Ressalta-se que tais inferências sobressaem com base nas médias estimadas, tendo-se em vista que os desvios padrão relativamente altos devem-se ao fato de ter sido considerado, no processo de simulação, um elevado percentual de dispersão para a característica (20\%), visando a representar possíveis outliers.

Para os acasalamentos ao acaso e entre os melhores, notou-se relativa correspondência no progresso fenotípico, com preeminência do segundo acasalamento, em tamanhos de família com 10, 20, 30 e 40 descendentes (Tabela 2). Em famílias com 50 descendentes, maior similaridade pôde ser observada entre as duas estratégias, com hegemonia do acasalamento aleatório, na maioria das gerações.

O poder de detecção de locos quantitativos está relacionado com o número de indivíduos e sua variabilidade genética. Acasalamentos que envolvam indivíduos contrastantes, a exemplo da distribuição dos extremos, contribuem para melhorias nos programas de seleção (Bhering \& Cruz 2008).

Segundo Zhao et al. (2012), Lu et al. (2013) e Nishio et al. (2013), é aceitável que a aplicação da distribuição dos extremos, no acasalamento seletivo, proporcione melhores resultados para a característica sobre a qual os indivíduos foram selecionados, por favorecer a divergência genotípica. Os indivíduos geneticamente divergentes são os mais informativos para a detecção de locos quantitativos. Na distribuição normal, para determinado caráter quantitativo, as progênies com valores fenotípicos de mais de um desvio padrão da média representam, aproximadamente, $33 \%$ de toda a população. Este percentual contribui com $81 \%$ de toda a informação necessária para a ligação de marcador e locos quantitativos (Van Gestel et al. 2000). Em contrapartida, indivíduos centrados próximos à média fenotípica da população pouco contribuem.

$\mathrm{O}$ acasalamento seletivo reduz o número de gerações para se obterem incrementos fenotípicos similares aos alcançados com os acasalamentos aleatórios e entre os melhores (Tabela 2). Ele também possibilita melhores ganhos fenotípicos, quando se comparam famílias de diferentes tamanhos, pois valores fenotípicos obtidos nas gerações finais, ao adotar o acasalamento seletivo em famílias com $10 \mathrm{ou}$ 20 descendentes, foram superiores aos incrementos alcançados com as outras duas estratégias de acasalamento, admitindo-se, contudo, famílias com 40 ou 50 descendentes (Tabela 2). Este fato evidencia a capacidade de a distribuição dos extremos reduzir o tamanho da população (número de indivíduos), em programas de melhoramento, sem comprometer o progresso genético (Jangarelli \& Euclydes 2010).

Sete grupos distintos foram formados pelo método de Tocher (Tabela 3). No primeiro grupo, 
Tabela 2. Valores fenotípicos médios e desvios padrão, ao longo de 20 gerações sob seleção assistida por marcadores (Seropédica, RJ, 2012).

\begin{tabular}{|c|c|c|c|c|c|c|c|c|c|c|c|c|}
\hline \multirow{2}{*}{$\mathrm{T}$} & & & \multicolumn{10}{|c|}{ Geração } \\
\hline & & & 1 & 2 & 3 & 4 & 5 & 6 & 7 & 8 & 9 & 10 \\
\hline 1 & \multirow{3}{*}{10} & AA & $11,67 \pm 0,19$ & $12,33 \pm 0,26$ & $12,76 \pm 0,32$ & $13,38 \pm 0,39$ & $14,00 \pm 0,46$ & $14,53 \pm 0,61$ & $15,12 \pm 0,65$ & $15,70 \pm 0,79$ & $16,16 \pm 0,79$ & $16,58 \pm 0,86$ \\
\hline 2 & & ИM & $1,68 \pm 0,19$ & $12,24 \pm 0,29$ & $12,80 \pm 0,34$ & $13,46 \pm 0,37$ & $14,12 \pm 0,47$ & $14,73 \pm 0,42$ & $15,25 \pm 0,40$ & $15,87 \pm 0,48$ & $16,35 \pm 0,51$ & $16,87 \pm 0,65$ \\
\hline 3 & & MP & $11,62 \pm 0,23$ & $12,59 \pm 0,31$ & $13,33 \pm 0,43$ & $13,98 \pm 0,58$ & $14,65 \pm 0,59$ & $15,23 \pm 0,64$ & $15,97 \pm 0,61$ & $16,49 \pm 0,72$ & $16,89 \pm 0,69$ & $17,26 \pm 0,65$ \\
\hline 4 & \multirow{3}{*}{20} & AA & $11,72 \pm 0,13$ & $12,29 \pm 0,33$ & $13,15 \pm 0,28$ & $14,01 \pm 0,44$ & $14,69 \pm 0,52$ & $15,40 \pm 0,61$ & $16,11 \pm 0,70$ & $16,62 \pm 0,70$ & $17,07 \pm 0,69$ & $17,37 \pm 0,82$ \\
\hline 5 & & MM & $11,70 \pm 0,16$ & $12,39 \pm 0,26$ & $13,21 \pm 0,31$ & $13,97 \pm 0,28$ & $14,57 \pm 0,51$ & $15,28 \pm 0,67$ & $16,03 \pm 0,63$ & $16,47 \pm 0,66$ & $17,04 \pm 0,73$ & $17,41 \pm 0,81$ \\
\hline 6 & & MP & $11,68 \pm 0,16$ & $12,88 \pm 0,24$ & $13,85 \pm 0,34$ & $14,73 \pm 0,45$ & $15,59 \pm 0,47$ & $16,24 \pm 0,61$ & $16,94 \pm 0,70$ & $17,42 \pm 0,82$ & $17,94 \pm 0,86$ & $18,31 \pm 1,00$ \\
\hline 7 & \multirow{3}{*}{30} & AA & $11,64 \pm 0,10$ & $12,28 \pm 0,23$ & $13,19 \pm 0,36$ & $14,10 \pm 0,46$ & $14,82 \pm 0,44$ & $15,43 \pm 0,43$ & $16,04 \pm 0,53$ & $16,69 \pm 0,56$ & $17,10 \pm 0,67$ & $17,33 \pm 0,81$ \\
\hline 8 & & MM & $11,65 \pm 0,11$ & $12,36 \pm 0,21$ & $13,21 \pm 0,38$ & $14,30 \pm 0,38$ & $15,09 \pm 0,51$ & $15,77 \pm 0,55$ & $16,48 \pm 0,57$ & $17,06 \pm 0,55$ & $17,44 \pm 0,75$ & $17,72 \pm 0,84$ \\
\hline 9 & & MP & $11,65 \pm 0,10$ & $12,89 \pm 0,34$ & $14,19 \pm 0,45$ & $15,15 \pm 0,41$ & $15,98 \pm 0,52$ & $16,70 \pm 0,49$ & $17,32 \pm 0,60$ & $17,86 \pm 0,61$ & $18,27 \pm 0,56$ & $18,69 \pm 0,58$ \\
\hline 10 & \multirow{3}{*}{40} & AA & $11,66 \pm 0,09$ & $12,31 \pm 0,24$ & $13,36 \pm 0,43$ & $14,18 \pm 0,52$ & $14,89 \pm 0,66$ & $15,58 \pm 0,77$ & $16,07 \pm 0,79$ & $16,58 \pm 0,89$ & $17,04 \pm 0,92$ & $17,29 \pm 0,90$ \\
\hline 11 & & MM & $11,66 \pm 0,14$ & $12,41 \pm 0,19$ & $13,50 \pm 0,46$ & $14,45 \pm 0,60$ & $15,32 \pm 0,65$ & $16,02 \pm 0,69$ & $16,62 \pm 0,66$ & $17,03 \pm 0,76$ & $17,46 \pm 0,82$ & $17,82 \pm 0,93$ \\
\hline 12 & & MP & $11,65 \pm 0,10$ & $12,87 \pm 0,41$ & $14,21 \pm 0,60$ & $15,12 \pm 0,67$ & $16,12 \pm 0,76$ & $16,86 \pm 0,74$ & $17,44 \pm 0,68$ & $17,97 \pm 0,69$ & $18,42 \pm 0,85$ & $18,75 \pm 0,84$ \\
\hline 13 & \multirow{3}{*}{50} & AA & $11,65 \pm 0,08$ & $12,43 \pm 0,16$ & $13,56 \pm 0,32$ & $14,49 \pm 0,43$ & $15,27 \pm 0,46$ & $16,04 \pm 0,48$ & $16,58 \pm 0,66$ & $17,11 \pm 0,66$ & & $17,88 \pm 0,96$ \\
\hline 14 & & MM & $11,63 \pm 0,08$ & $12,35 \pm 0,25$ & $13,49 \pm 0,53$ & $14,45 \pm 0,69$ & $15,31 \pm 0,80$ & $16,03 \pm 0,95$ & $16,62 \pm 1,15$ & $17,07 \pm 1,26$ & $17,46 \pm 1,36$ & $17,84 \pm 1,38$ \\
\hline 15 & & MP & $11,66 \pm 0,07$ & & & & $15,96 \pm 0,73$ & $16,72 \pm 0,66$ & $17,32 \pm 0,77$ & $17,87 \pm 0,75$ & & \\
\hline \multirow{2}{*}{$\mathrm{T}$} & \multirow{2}{*}{$\mathrm{TF}$} & & \multicolumn{10}{|c|}{ Geração } \\
\hline & & & 11 & 12 & 13 & 14 & 15 & 16 & 17 & 18 & 19 & 20 \\
\hline 1 & \multirow{3}{*}{10} & AA & $17,04 \pm 0,77$ & $17,51 \pm 0,90$ & $17,92 \pm 0,96$ & $18,16 \pm 0,99$ & $18,36 \pm 1,06$ & $18,70 \pm 1,04$ & $19,01 \pm 1,00$ & $19,11 \pm 0,87$ & $19,24 \pm 0,89$ & $19,36 \pm$ \\
\hline 2 & & MM & $17,44 \pm 0,75$ & $17,92 \pm 0,77$ & $18,11 \pm 0,75$ & $18,37 \pm 0,78$ & $18,64 \pm 0,82$ & $19,09 \pm 0,72$ & $19,23 \pm 0,77$ & $19,39 \pm 0,73$ & $19,52 \pm 0,78$ & $19,61 \pm 0,78$ \\
\hline 3 & & MP & $17,72 \pm 0,80$ & $18,05 \pm 0,70$ & $18,32 \pm 0,78$ & $18,70 \pm 0,83$ & $18,91 \pm 0,76$ & $19,21 \pm 0,86$ & $19,40 \pm 0,83$ & $19,60 \pm 0,87$ & $19,79 \pm 0,85$ & $20,00 \pm 0,91$ \\
\hline 4 & \multirow{3}{*}{20} & AA & $17,65 \pm 0,84$ & $18,00 \pm 0,90$ & $18,04 \pm 0,98$ & $18,23 \pm 0,96$ & $18,39 \pm 1,08$ & $18,52 \pm 1,14$ & $18,66 \pm 1,15$ & $18,72 \pm 1,22$ & $18,73 \pm 1,20$ & $18,73 \pm 1,18$ \\
\hline 5 & & MM & $17,72 \pm 0,96$ & $18,17 \pm 0,98$ & $18,25 \pm 0,96$ & $18,36 \pm 1,03$ & $18,53 \pm 1,03$ & $18,81 \pm 1,08$ & $19,01 \pm 1,05$ & $19,03 \pm 1,11$ & $19,14 \pm 1,20$ & $19,14 \pm 1,23$ \\
\hline 6 & & MP & $18,56 \pm 1,04$ & $18,87 \pm 1,06$ & $19,12 \pm 1,11$ & $19,32 \pm 1,09$ & $19,35 \pm 1,15$ & $19,48 \pm 1,09$ & $19,54 \pm 1,18$ & $19,58 \pm 1,19$ & $19,70 \pm 1,23$ & $19,73 \pm 1,18$ \\
\hline 7 & \multirow{3}{*}{30} & AA & $17,73 \pm 0,88$ & $18,01 \pm 1,00$ & $18,10 \pm 1,15$ & $18,19 \pm 1,22$ & $18,32 \pm 1,32$ & $18,39 \pm 1,32$ & $18,47 \pm 1,39$ & $18,53 \pm 1,41$ & $18,62 \pm 1,43$ & $18,53 \pm 1,43$ \\
\hline 8 & & MM & $18,22 \pm 0,96$ & $18,51 \pm 0,94$ & $18,68 \pm 0,98$ & $18,79 \pm 1,00$ & $18,89 \pm 1,01$ & $19,00 \pm 1,11$ & $19,01 \pm 1,09$ & $19,06 \pm 1,14$ & $19,04 \pm 1,20$ & $19,06 \pm 1,25$ \\
\hline 9 & & MP & $19,05 \pm 0,62$ & $19,35 \pm 0,67$ & $19,53 \pm 0,67$ & $19,69 \pm 0,72$ & $19,85 \pm 0,78$ & $19,90 \pm 0,76$ & $19,99 \pm 0,78$ & $20,03 \pm 0,79$ & $20,07 \pm 0,80$ & $20,10 \pm 0,79$ \\
\hline 10 & \multirow{3}{*}{40} & AA & $17,47 \pm 0,92$ & $17,70 \pm 0,95$ & $17,80 \pm 1,04$ & $17,87 \pm 0,96$ & $18,02 \pm 1,02$ & $18,08 \pm 1,01$ & $18,13 \pm 0,98$ & $18,18 \pm 1,00$ & $18,19 \pm 1,02$ & $18,19 \pm 0,98$ \\
\hline 11 & & MM & $18,07 \pm 0,92$ & $18,35 \pm 0,91$ & $18,46 \pm 0,99$ & $18,51 \pm 1,03$ & $18,64 \pm 1,04$ & $18,71 \pm 0,97$ & $18,75 \pm 0,99$ & $18,78 \pm 1,01$ & $18,78 \pm 0,99$ & $18,79 \pm 1,03$ \\
\hline 12 & & MP & $18,92 \pm 0,90$ & $19,12 \pm 1,02$ & $19,31 \pm 0,86$ & $19,39 \pm 0,91$ & $19,46 \pm 0,86$ & $19,53 \pm 0,93$ & $19,62 \pm 0,92$ & $19,65 \pm 0,94$ & $19,66 \pm 0,92$ & $19,67 \pm 0,93$ \\
\hline 13 & \multirow{3}{*}{50} & AA & $18,11 \pm 1,10$ & $18,37 \pm 1,23$ & $18,52 \pm 1,27$ & $18,54 \pm 1,33$ & $18,60 \pm 1,37$ & $18,62 \pm 1,42$ & $18,62 \pm 1,42$ & $18,65 \pm 1,48$ & $18,67 \pm 1,45$ & $18,68 \pm 1,41$ \\
\hline 14 & & MM & $18,13 \pm 1,39$ & $18,29 \pm 1,43$ & $18,34 \pm 1,55$ & $18,39 \pm 1,57$ & $18,45 \pm 1,65$ & $18,50 \pm 1,68$ & $18,52 \pm 1,60$ & $18,54 \pm 1,60$ & $18,54 \pm 1,62$ & $18,56 \pm 1,69$ \\
\hline 15 & & MP & $18,66 \pm 1,04$ & $18,84 \pm 1,08$ & $18,97 \pm 1,04$ & $19,05 \pm 1,07$ & $19,09 \pm 1,14$ & $19,16 \pm 1,14$ & $19,18 \pm 1,13$ & $19,20 \pm 1,10$ & $19,24 \pm 1,12$ & $19,26 \pm 1,09$ \\
\hline
\end{tabular}

T: tratamento; TF: tamanho da família; EA: estratégia de acasalamento (AA: acasalamento ao acaso; MM: acasalamento melhores vs. melhores; MP: acasalamento seletivo - distribuição dos extremos).

Tabela 3. Grupos, limite e distâncias obtidas entre os tratamentos (estratégias de acasalamento), pela aplicação do método de otimização de Tocher, para o parâmetro valor fenotípico (Seropédica, RJ, 2012).

\begin{tabular}{ccccl}
\hline $\begin{array}{c}\text { Número } \\
\text { do grupo }\end{array}$ & $\begin{array}{c}\text { Limite } \\
(\theta)\end{array}$ & $\begin{array}{c}\text { Distância } \\
\text { obtida }\end{array}$ & $\begin{array}{c}\text { Número de } \\
\text { tratamentos }\end{array}$ & $\begin{array}{l}\text { Tratamentos } \\
\text { pertencentes }\end{array}$ \\
\hline 1 & 0,3286 & 0,07 & 2 & $11 ; 13$ \\
1 & 0,3286 & 0,11 & 3 & $11 ; 13 ; 14$ \\
1 & 0,3286 & 0,26 & 4 & $11 ; 13 ; 14 ; 8$ \\
\hline 2 & 0,3286 & 0,10 & 2 & $4 ; 7$ \\
2 & 0,3286 & 0,25 & 3 & $4 ; 7 ; 5$ \\
\hline 3 & 0,3286 & 0,24 & 2 & $1 ; 2$ \\
\hline 4 & 0,3286 & 0,25 & 2 & $9 ; 12$ \\
\hline 5 & 0,3286 & 0,32 & 2 & $6: 15$ \\
\hline 6 & 0,3286 & 0,81 & 1 & 3 \\
\hline 7 & 0,3286 & - & 1 & 10 \\
\hline
\end{tabular}

foram classificados os tratamentos $8,11,13$ e 14 . O segundo grupo foi formado pelos tratamentos $4,5 \mathrm{e}$ 7. Os grupos três, quatro e cinco foram constituídos, cada um, por duas estratégias de acasalamento: 1 e 2; 9 e 12; e 6 e 15, respectivamente. Já os grupos seis e sete compreenderam apenas um tratamento: 3 e 10, respectivamente.

Evidenciou-se, com base nos valores fenotípicos médios, a inferioridade dos grupos dois e três e a superioridade dos grupos quatro e cinco. Ressalta-se que os grupos que otimizaram os ganhos fenotípicos foram constituídos apenas por tratamentos que representavam estratégias envolvendo o acasalamento seletivo, em diferentes tamanhos de família (trata- 
mentos 6, 9, 12 e 15). Mesmo em tamanhos pequenos, como é o caso de famílias com 10 descendentes, o acasalamento seletivo (tratamento 3 ) foi classificado de maneira distinta das outras duas estratégias (tratamentos 1 e 2), o que corrobora a supremacia da distribuição dos extremos, no sentido de potencializar o progresso fenotípico, em um programa de melhoramento genético assistido por marcadores.

O resultado estabelecido pelo método hierárquico da ligação completa, por meio do dendrograma, diverge, em relação ao método de Tocher, por não estabelecer um número ótimo de grupos, ilustrando apenas ramificações para descrições a respeito dos padrões de similaridade (Figura 1). Todavia, delimitações para a formação de determinado número de grupos podem ser definidas.

A distância, em percentagem, de $25 \%$ foi o nível adotado para o ponto de corte, com o propósito de manter o mesmo número de grupos propostos pelo método de otimização (sete grupos) e, em seguida, descrever semelhanças, no que diz respeito à constituição destes grupos, entre os dois métodos.

Os resultados demonstraram correspondência entre os métodos hierárquico e de otimização, com relação à composição dos grupos, os quais foram constituídos pelas mesmas estratégias de acasalamento (tratamentos). Os métodos de agrupamento apresentam fundamentações teóricas distintas, recomendando sua aplicação em metodologias diferentes, com o propósito de analogia e validação dos resultados, o que justifica a aplicação dos dois métodos. As análises convalidam as suposições acerca da supremacia da distribuição dos extremos, na otimização do incremento fenotípico e redução

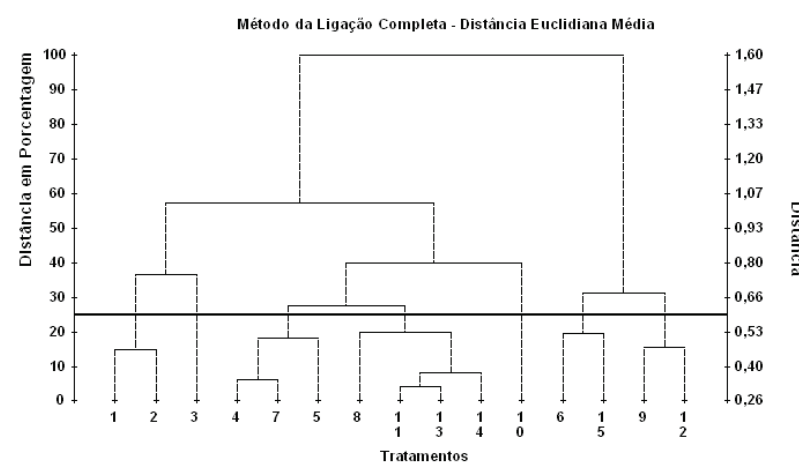

Figura 1. Dendrograma do método hierárquico da ligação completa, pela análise de 15 tratamentos (estratégias de acasalamento), para o parâmetro valor fenotípico (Seropédica, RJ, 2012). do tamanho da população requerida em programas de melhoramento.

$\mathrm{O}$ acasalamento seletivo (MP) proporcionou menor média endogâmica em todos os tamanhos de família admitidos, o que favorece maior variabilidade genética, ao longo das gerações sob seleção assistida por marcadores (Tabela 4). Relativa equivalência nas médias endogâmicas foi observada entre as outras duas estratégias (AA e MM), tendo o acasalamento entre os melhores (MM) maior capacidade em retardar o acréscimo endogâmico. Correspondência mais eminente entre estas duas estratégias foi observada nas famílias com 40 e 50 descendentes.

A principal consequência da endogamia é o fenômeno conhecido como depressão endogâmica, caracterizado pela redução do valor médio fenotípico (Cunha et al. 2004). Os coeficientes de endogamia de menores magnitudes estão relacionados aos maiores valores fenotípicos, de acordo com o tamanho de família e o tipo de acasalamento (Tabelas 2 e 4). O acasalamento estratégico que utiliza a distribuição dos extremos retarda a consanguinidade entre os indivíduos e, indiretamente, mantém níveis mais altos de variabilidade genética. Este fato maximiza a detecção de locos quantitativos por marcadores, em uma seleção assistida, o que incrementa os ganhos fenotípicos, ao longo das gerações sob seleção assistida por marcadores (Tabela 2).

Para o parâmetro endogamia, o método de otimização de Tocher formou cinco grupos (Tabela 5). No primeiro grupo, foram classificados os tratamentos 1,2 e 3 . O segundo grupo foi formado pelos tratamentos 7, 8, 11, 12 e 15 . O terceiro compreendeu as estratégias assinaladas pelos tratamentos 10,13 e 14 . Já o grupo quatro foi constituído pelos tratamentos 4, 5 e 9 , ficando apenas o tratamento 6 no grupo cinco.

$\mathrm{O}$ coeficiente de endogamia aumenta à medida em que o número de indivíduos aparentados se eleva. Famílias com maior número de descendentes assinalam maior média endogâmica, ao passo que, em famílias pequenas, o incremento endogâmico tende a ser mais moderado.

Exceto para o tamanho de família com 10 descendentes, em que as três estratégias constituíram o mesmo grupo, para os demais tamanhos, o acasalamento seletivo mostrou-se eficiente, na tentativa de retardar o acréscimo endogâmico. Considerando-se famílias com 20 descendentes, a estratégia seletiva diferiu das outras duas, constituindo, exclusivamente, o grupo cinco, que sinalizava menores médias 
Tabela 4. Endogamia média e desvios padrão, ao longo de 20 gerações, sob seleção assistida por marcadores (Seropédica, RJ, 2012).

\begin{tabular}{|c|c|c|c|c|c|c|c|c|c|c|c|c|}
\hline \multirow{2}{*}{$\mathrm{T}$} & \multirow{2}{*}{ TF } & \multirow{2}{*}{ EA } & \multicolumn{10}{|c|}{ Geração } \\
\hline & & & 1 & 2 & 3 & 4 & 5 & 6 & 7 & 8 & 9 & 10 \\
\hline 1 & & $\mathrm{AA}$ & $0,075 \pm, 036$ & $0,093 \pm, 065$ & $0,168 \pm, 054$ & $0,233 \pm, 053$ & $0,280 \pm, 045$ & $0,335 \pm, 058$ & $0,385 \pm, 066$ & $0,430 \pm, 066$ & $0,482 \pm, 064$ & $0,508 \pm, 060$ \\
\hline 2 & 10 & $\mathrm{MM}$ & $0,076 \pm, 024$ & $0,093 \pm, 041$ & $0,169 \pm, 048$ & $0,212 \pm, 052$ & $0,271 \pm, 051$ & $0,335 \pm, 058$ & $0,376 \pm, 061$ & $0,429 \pm, 065$ & $0,471 \pm, 063$ & $0,503 \pm, 047$ \\
\hline 3 & & MP & $0,025 \pm, 014$ & $0,057 \pm, 033$ & $0,145 \pm, 038$ & $0,205 \pm, 046$ & $0,258 \pm, 046$ & $0,314 \pm, 055$ & $0,370 \pm, 056$ & $0,416 \pm, 059$ & $0,459 \pm, 057$ & $0,488 \pm, 058$ \\
\hline 4 & & AA & $0,076 \pm, 049$ & $0,184 \pm, 092$ & $0,289 \pm, 101$ & $0,368 \pm, 103$ & $0,423 \pm, 093$ & $0,481 \pm, 090$ & $0,540 \pm, 088$ & $0,591 \pm, 082$ & $0,646 \pm, 079$ & $0,677 \pm, 078$ \\
\hline 5 & 20 & $\mathrm{MM}$ & $0,077 \pm, 032$ & $0,201 \pm, 070$ & $0,308 \pm, 089$ & $0,376 \pm, 090$ & $0,442 \pm, 100$ & $0,487 \pm, 089$ & $0,536 \pm, 087$ & $0,587 \pm, 068$ & $0,625 \pm, 061$ & $0,662 \pm, 055$ \\
\hline 6 & & MP & $0,027 \pm, 019$ & $0,141 \pm, 046$ & $0,251 \pm, 054$ & $0,320 \pm, 078$ & $0,397 \pm, 065$ & $0,455 \pm, 064$ & $0,508 \pm, 070$ & $0,557 \pm, 069$ & $0,600 \pm, 072$ & $0,634 \pm, 073$ \\
\hline 7 & & $\mathrm{AA}$ & $0,077 \pm, 043$ & $0,267 \pm, 091$ & $0,356 \pm, 102$ & $0,434 \pm, 092$ & $0,491 \pm, 106$ & $0,564 \pm, 088$ & $0,606 \pm, 086$ & $0,654 \pm, 073$ & $0,695 \pm, 067$ & $0,716 \pm, 064$ \\
\hline 8 & 30 & $\mathrm{MM}$ & $0,079 \pm, 045$ & $0,226 \pm, 090$ & $0,311 \pm, 081$ & $0,375 \pm, 103$ & $0,456 \pm, 080$ & $0,517 \pm, 067$ & $0,577 \pm, 071$ & $0,638 \pm, 063$ & $0,673 \pm, 052$ & $0,712 \pm, 054$ \\
\hline 9 & & MP & $0,028 \pm, 020$ & $0,197 \pm, 053$ & $0,272 \pm, 054$ & $0,362 \pm, 071$ & $0,416 \pm, 069$ & $0,482 \pm, 063$ & $0,540 \pm, 050$ & $0,592 \pm, 059$ & $0,627 \pm, 063$ & $0,679 \pm, 041$ \\
\hline 10 & & $\mathrm{AA}$ & $0,152 \pm, 018$ & $0,360 \pm, 037$ & $0,432 \pm, 032$ & $0,503 \pm, 045$ & $0,557 \pm, 046$ & $0,615 \pm, 044$ & $0,653 \pm, 042$ & $0,694 \pm, 036$ & $0,727 \pm, 051$ & $0,759 \pm, 044$ \\
\hline 11 & 40 & $\mathrm{MM}$ & $0,149 \pm, 039$ & $0,295 \pm, 082$ & $0,364 \pm, 083$ & $0,436 \pm, 078$ & $0,505 \pm, 063$ & $0,573 \pm, 061$ & $0,616 \pm, 062$ & $0,671 \pm, 044$ & $0,712 \pm, 054$ & $0,745 \pm, 058$ \\
\hline 12 & & MP & $0,048 \pm, 018$ & $0,214 \pm, 042$ & $0,287 \pm, 046$ & $0,374 \pm, 065$ & $0,473 \pm, 064$ & $0,536 \pm, 065$ & $0,589 \pm, 053$ & $0,646 \pm, 038$ & $0,691 \pm, 049$ & $0,719 \pm, 040$ \\
\hline 13 & & AA & $0,151 \pm, 037$ & $0,312 \pm, 075$ & $0,398 \pm, 059$ & $0,449 \pm, 057$ & $0,522 \pm, 058$ & $0,581 \pm, 072$ & $0,629 \pm, 047$ & $0,671 \pm, 048$ & $0,710 \pm, 049$ & $0,751 \pm, 044$ \\
\hline 14 & 50 & $\mathrm{MM}$ & $0,147 \pm, 042$ & $0,298 \pm, 079$ & $0,414 \pm, 071$ & & $0,535 \pm, 053$ & $0,601 \pm, 052$ & $0,647 \pm, 049$ & $0,679 \pm, 045$ & $0,717 \pm, 046$ & $0,752 \pm, 039$ \\
\hline 15 & & MP & $0,049 \pm, 019$ & $0,210 \pm, 046$ & $0,290 \pm, 042$ & $0,401 \pm, 050$ & $0,476 \pm, 045$ & $0,549 \pm, 048$ & $0,597 \pm, 049$ & $0,649 \pm, 041$ & $0,693 \pm, 045$ & $0,728 \pm, 042$ \\
\hline \multirow{2}{*}{$\mathrm{T}$} & & \multicolumn{11}{|c|}{ Geração } \\
\hline & $1 \mathrm{~F}$ & & 11 & 12 & 13 & 14 & 15 & 16 & 17 & 18 & 19 & 20 \\
\hline 1 & & $\mathrm{AA}$ & $0,544 \pm, 063$ & $0,583 \pm, 062$ & $0,615 \pm, 061$ & $0,646 \pm, 067$ & $0,677 \pm, 073$ & $0,690 \pm, 076$ & $0,718 \pm, 074$ & $0,748 \pm, 078$ & $0,774 \pm, 074$ & $0,800 \pm, 067$ \\
\hline 2 & 10 & $\mathrm{MM}$ & $0,544 \pm, 057$ & $0,585 \pm, 057$ & $0,616 \pm, 060$ & $0,651 \pm, 063$ & $0,678 \pm, 062$ & $0,694 \pm, 052$ & $0,714 \pm, 053$ & $0,747 \pm, 053$ & $0,763 \pm, 049$ & $0,780 \pm, 046$ \\
\hline 3 & & MP & $0,526 \pm, 057$ & $0,562 \pm, 060$ & $0,595 \pm, 057$ & $0,616 \pm, 057$ & $0,649 \pm, 055$ & $0,672 \pm, 054$ & $0,690 \pm, 058$ & $0,706 \pm, 058$ & $0,730 \pm, 053$ & $0,747 \pm, 049$ \\
\hline 4 & & AA & $0,711 \pm, 077$ & $0,745 \pm, 070$ & $0,768 \pm, 061$ & $0,800 \pm, 058$ & $0,829 \pm, 059$ & $0,853 \pm, 056$ & $0,868 \pm, 059$ & $0,887 \pm, 058$ & $0,905 \pm, 050$ & $0,922 \pm, 041$ \\
\hline 5 & 20 & MM & $0,694 \pm, 051$ & $0,725 \pm, 050$ & $0,751 \pm, 056$ & $0,781 \pm, 056$ & $0,799 \pm, 055$ & $0,825 \pm, 049$ & $0,844 \pm, 047$ & $0,865 \pm, 048$ & $0,889 \pm, 041$ & $0,904 \pm, 038$ \\
\hline 6 & & MP & $0,667 \pm, 083$ & $0,694 \pm, 073$ & $0,717 \pm, 068$ & $0,741 \pm, 063$ & $0,766 \pm, 068$ & $0,784 \pm, 054$ & $0,809 \pm, 058$ & $0,827 \pm, 052$ & $0,845 \pm, 053$ & $0,862 \pm, 046$ \\
\hline 7 & & $\mathrm{AA}$ & $0,753 \pm, 057$ & $0,779 \pm, 052$ & $0,810 \pm, 055$ & $0,844 \pm, 050$ & $0,864 \pm, 043$ & $0,893 \pm, 045$ & $0,909 \pm, 044$ & $0,928 \pm, 035$ & $0,940 \pm, 030$ & $0,951 \pm, 024$ \\
\hline 8 & 30 & $\mathrm{MM}$ & $0,741 \pm, 057$ & $0,776 \pm, 055$ & $0,808 \pm, 047$ & $0,833 \pm, 044$ & $0,861 \pm, 049$ & $0,880 \pm, 050$ & $0,897 \pm, 042$ & $0,915 \pm, 047$ & $0,930 \pm, 040$ & $0,944 \pm, 034$ \\
\hline 9 & & MP & $0,711 \pm, 043$ & $0,738 \pm, 045$ & $0,762 \pm, 044$ & $0,796 \pm, 046$ & $0,821 \pm, 045$ & $0,842 \pm, 048$ & $0,866 \pm, 046$ & $0,885 \pm, 044$ & $0,902 \pm, 038$ & $0,918 \pm, 036$ \\
\hline 10 & & $\mathrm{AA}$ & $0,792 \pm, 043$ & $0,821 \pm, 042$ & $0,850 \pm, 043$ & $0,868 \pm, 043$ & $0,897 \pm, 035$ & $0,918 \pm, 024$ & $0,931 \pm, 022$ & $0,944 \pm, 017$ & $0,955 \pm, 014$ & $0,964 \pm, 012$ \\
\hline 11 & 40 & MM & $0,768 \pm, 062$ & $0,799 \pm, 050$ & $0,829 \pm, 041$ & $0,857 \pm, 043$ & $0,881 \pm, 043$ & $0,901 \pm, 037$ & $0,917 \pm, 034$ & $0,933 \pm, 031$ & $0,946 \pm, 022$ & $0,956 \pm, 020$ \\
\hline 12 & & MP & $0,759 \pm, 047$ & $0,779 \pm, 044$ & $0,812 \pm, 043$ & $0,835 \pm, 041$ & $0,860 \pm, 039$ & $0,878 \pm, 035$ & $0,894 \pm, 035$ & $0,911 \pm, 032$ & $0,925 \pm, 031$ & $0,938 \pm, 026$ \\
\hline 13 & & AA & $0,790 \pm, 044$ & $0,820 \pm, 045$ & $0,850 \pm, 042$ & $0,876 \pm, 045$ & $0,900 \pm, 039$ & $0,918 \pm, 033$ & $0,932 \pm, 032$ & $0,946 \pm, 023$ & $0,957 \pm, 018$ & $0,965 \pm, 014$ \\
\hline 14 & 50 & $\mathrm{MM}$ & $0,784 \pm, 052$ & $0,818 \pm, 045$ & $0,849 \pm, 042$ & $0,873 \pm, 038$ & $0,895 \pm, 034$ & $0,914 \pm, 029$ & $0,931 \pm, 023$ & $0,944 \pm, 018$ & $0,955 \pm, 014$ & $0,964 \pm, 012$ \\
\hline 15 & & MP & $0,760 \pm, 048$ & $0,797 \pm, 041$ & $0,824 \pm, 043$ & $0,843 \pm, 045$ & $0,870 \pm, 040$ & $0,891 \pm, 034$ & $0,907 \pm, 031$ & $0,920 \pm, 030$ & $0,935 \pm, 023$ & $0,946 \pm, 021$ \\
\hline
\end{tabular}

Tabela 5. Grupos, limite e distâncias obtidas entre os tratamentos (estratégias de acasalamento), pela aplicação do método de otimização de Tocher, para o parâmetro endogamia (Seropédica, RJ, 2012).

\begin{tabular}{ccccl}
\hline $\begin{array}{c}\text { Número } \\
\text { do grupo }\end{array}$ & $\begin{array}{c}\text { Limite } \\
(\theta)\end{array}$ & $\begin{array}{c}\text { Distância } \\
\text { obtida }\end{array}$ & $\begin{array}{c}\text { Número de } \\
\text { tratamentos }\end{array}$ & $\begin{array}{l}\text { Tratamentos } \\
\text { pertencentes }\end{array}$ \\
\hline 1 & 0,0401 & 0,01 & 2 & $1 ; 2$ \\
1 & 0,0401 & 0,03 & 3 & $1 ; 2 ; 3$ \\
\hline 2 & 0,0401 & 0,01 & 2 & $12 ; 15$ \\
2 & 0,0401 & 0,02 & 3 & $12 ; 15 ; 8$ \\
2 & 0,0401 & 0,03 & 4 & $12 ; 15 ; 8 ; 7$ \\
2 & 0,0401 & 0,04 & 5 & $12 ; 15 ; 8 ; 7 ; 11$ \\
\hline 3 & 0,0401 & 0,01 & 2 & $13 ; 14$ \\
3 & 0,0401 & 0,02 & 3 & $13 ; 14 ; 10$ \\
\hline 4 & 0,0401 & 0,01 & 2 & $4 ; 9$ \\
4 & 0,0401 & 0,02 & 3 & $4 ; 9 ; 5$ \\
\hline 5 & 0,0401 & - & 1 & 6 \\
\hline
\end{tabular}

endogâmicas, ao longo das gerações. Para 30, 40 e 50 descendentes, a distribuição dos extremos não só diferiu, mas, também, foi capaz de agrupar-se com estratégias que continham menor número de indivíduos (menor tamanho de família), o que demonstra favorecimento no retardo da consanguinidade, para as estratégias que compõem os grupos dois e quatro.

Evidenciou-se que a distribuição dos extremos permite a utilização racional da variabilidade genética, o que retarda o aumento da endogamia, mesmo em tamanhos de família maiores, sem comprometer o progresso fenotípico e genético da população sob seleção.

O dendrograma estabelecido pelo método hierárquico possibilitou delimitações, com a finalidade 


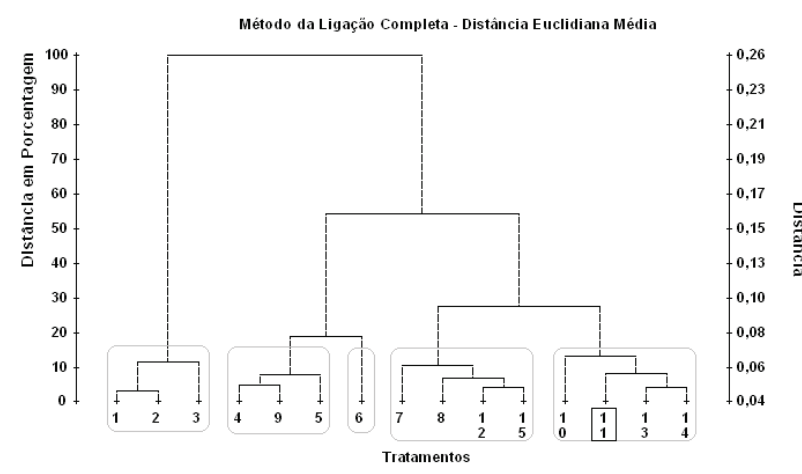

Figura 2. Dendrograma do método hierárquico da ligação completa, pela análise de 15 tratamentos (estratégias de acasalamento), para o parâmetro endogamia (Seropédica, RJ, 2012).

de manter o mesmo número de grupos propostos pelo método de Tocher (Figura 2). Correspondência de, aproximadamente, $93 \%$ foi verifica entre os métodos, no que diz respeito aos tratamentos representados em cada um dos cinco grupos. Apenas o tratamento onze (TF 40 e MM) agrupou-se em partições distintas, entre os métodos. A similaridade na constituição dos grupos entre os métodos corrobora a supremacia do acasalamento seletivo na capacidade de retardar o incremento endogâmico.

\section{CONCLUSÕES}

1. Os métodos de agrupamento de Tocher e da ligação completa mostraram-se válidos para caracterizar e distinguir a distribuição dos extremos (acasalamento seletivo - MP) das demais estratégias de acasalamento, conforme o tamanho de família admitido, na tentativa de otimizar o valor fenotípico e retardar a endogamia.

2. A aplicação do princípio da genotipagem seletiva em acasalamentos dirigidos evidenciou melhores incrementos fenotípicos e maior controle da endogamia, para a característica sob seleção assistida por marcadores.

\section{REFERÊNCIAS}

BHERING, L. L.; CRUZ, C. D. Tamanho de população ideal para mapeamento genético em famílias de irmãos completos. Pesquisa Agropecuária Brasileira, Brasília, DF, v. 43, n. 3, p. 379-385, 2008.

CUNHA, E. E. et al. Variabilidade genética e limite da seleção em populações de diferentes tipos de acasalamento.
Arquivo Brasileiro de Medicina Veterinária e Zootecnia, Belo Horizonte, v. 56, n. 2, p. 242-250, 2004.

EUCLYDES, R. F. Genesys: sistema de simulação genética. Viçosa: UFV, 2009. 1 CD-ROM.

ISHII, A. et al. Genome-wide association study for fatty acid composition in Japanese black cattle. Animal Science Journal, Urbana-Champaign, v. 84, n. 10, p. 675-682, 2013.

JACOMETO, C. B. et al. Variabilidade genética em tambaquis (Teleostei: Characidae) de diferentes regiões do Brasil. Pesquisa Agropecuária Brasileira, Brasília, DF, v. 45, n. 5, p. 481-487, 2010.

JANGARELLI, M. et al. Análise de agrupamento de diferentes densidades de marcadores no mapeamento genético por varredura genômica. Revista Ceres, Viçosa, v. 57, n. 6, p. 695-700, 2010.

JANGARELLI, M.; EUCLYDES, R. F. Otimização do tamanho de população sob acasalamento seletivo na seleção assistida por marcadores moleculares. Revista Brasileira de Zootecnia, Viçosa, v. 39, n. 12, p. 2625 2631, 2010.

LEÃO, G. F. M. et al. Melhoramento genético em zebuínos leiteiros: uma revisão. Agropecuária Científica no Semiárido, Campina Grande, v. 9, n. 4, p. 9-14, 2013.

LU, X. et al. Three EST-SSR markers associated with QTL for the growth of the clam Meretrix meretrix revealed by selective genotyping. Marine Biotechnology, New York, v. 15, n. 1, p. 16-25, 2013.

MALHADO, C. H. M. et al. Histórico genético e populacional do rebanho Nelore puro de origem no sertão nordestino. Pesquisa Agropecuária Brasileira, Brasília, DF, v. 44, n. 7, p. 713-718, 2009.

NEVES, H. H. R. et al. Acasalamento dirigido para aumentar a produção de animais geneticamente superiores e reduzir a variabilidade da progênie em bovinos. Revista Brasileira de Zootecnia, Viçosa, v. 38, n. 7, p. 1201-1204, 2009.

NISHIO, S. et al. Mapping and pedigree analysis of the gene that controls the easy peel pellicle trait in Japanese chestnut (Castanea crenata Sieb. et Zucc.). Tree Genetics \& Genomes, Berlin, v. 9, n. 3, p. 723-730, 2013.

REIS, R. L. et al. Estimativas de variância genética aditiva em populações selecionadas e não-selecionadas via simulação Monte Carlo utilizando o software R. Ciência e Agrotecnologia, Lavras, v. 33, n. 1, p. 285-291, 2009.

RIBEIRO JÚNIOR, J. I.; MELO, A. L. P. Guia prático para utilização do SAEG. Viçosa: UFV, 2008. 
RODRIGUES, J. I. S. et al. Mapeamento de QTL para conteúdos de proteína e óleo em soja. Pesquisa Agropecuária Brasileira, Brasília, DF, v. 45, n. 5, p. 472480, 2010.

ROSA, G. J. M. Delineamento de experimentos em genética genômica. Revista Brasileira de Zootecnia, Viçosa, v. 36, supl., p. 211-218, 2007.

SOUSA, K. R. S. et al. Mapeamento de locos de características quantitativas nos cromossomos 5,7 e 8 de suínos. Revista Brasileira de Zootecnia, Viçosa, v. 40, n. 1, p. 115-123, 2011.
VAN GESTEL, S. et al. Power of selective genotyping in genetic association analyses of quantitative traits. Behavior Genetics, New York, v. 30, n. 2, p. 141-146, 2000.

ZHAO, Y. et al. Impact of selective genotyping in the training population on accuracy and bias of genomic selection. Theoretical and Applied Genetics, Berlin, v. 125, n. 4, p. 707-713, 2012.

WRIGHT, S. Evolution in Mendelian population. Genetics, Maryland, v. 16, n. 2, p. 97-159, 1931. 